\title{
Bovine colostrum is a health food supplement which prevents NSAID induced gut damage
}

R J Playford, D N Floyd, C E Macdonald, D P Calnan, R O Adenekan, W Johnson, R A Goodlad, T Marchbank includes peptic ulceration and injury to the small and large intestine causing increased permeability with protein and blood loss and stricture formation. ${ }^{1-3}$

These pathologies often develop without symptoms and are of particular significance in the elderly where NSAIDs are often used for several years. While acid suppressants and prostaglandin analogues are of proven benefit for the prophylaxis of NSAID induced peptic ulceration, they are relatively ineffective in preventing small and large intestinal injury. ${ }^{3}$ In addition, use of prostaglandin analogues can be complicated by diarrhoea and they are contraindicated in young women because of their proabortive and teratogenic activity. ${ }^{4}$ Novel therapeutic approaches are therefore required.

Colostrum is the milk produced for the first few days after birth and is a rich natural source of nutrients, antibodies, and growth factors for the suckling neonate. Some studies suggest it may be of value in eliminating infection and stimulating growth of the neonatal gastrointestinal tract. ${ }^{5}{ }^{6}$ Its value in the prevention and treatment of adult gastrointestinal injury is, however, largely unexplored. We therefore examined the potential value of a defatted bovine colostral preparation to prevent gastric and small intestinal injury induced by the NSAID indomethacin. factor $\beta$ added at a dose similar to that found in the colostrum preparation $(12.5$ ng/rat), reduced injury by about $60 \%$.

Scientific Hospital Supplies International Ltd, 100 Wavertree

Boulevard, Liverpool

L7 9PT, UK

W Johnson

Correspondence to: Professor Playford.

Accepted for publication 13 January 1999 Addition of colostrum to drinking water $(10 \%$ vol/vol) prevented villus shortening in the mouse model of small intestinal injury. Addition of milk preparation was ineffective. Colostrum increased proliferation and cell migration of RIE-1 and HT-29 cells. These effects were mainly due to constituents of the colostrum with molecular weights greater than $30 \mathrm{kDa}$. Conclusions-Bovine colostrum could provide a novel, inexpensive approach for the prevention and treatment of the injurious effects of NSAIDs on the gut and may also be of value for the treatment of other ulcerative conditions of the bowel. (Gut 1999;44:653-658)

Keywords: gastrointestinal tract; intestinal injury; repair; nutrition

\section{Methods}

PREPARATION OF COLOSTRUM AND MILK FRACTIONS

Colostrum and milk solutions for use in the studies were prepared by Viable Bioproducts, Turku, Finland. The initial colostrum and milk whey solutions were passed through a microfilter $(0.2 \mu \mathrm{m}$ pore $)$. The final solutions were free of fat and lactose and were reduced in most of the major proteins including casein and lactalbumin, with the remaining protein being relatively rich in immunoglobulins and growth factors. This form of colostrum is commercially available as a health food supplement in the USA, UK, and the rest of Europe, and is marketed as a general "health promoting" product, particularly suitable for athletes. The total protein content of the colostrum and milk preparations were $4.3 \mathrm{mg} / \mathrm{ml}$. The concentrations of the various growth factors present in the colostrum preparation are incompletely defined but include: insulin like growth factor I

anti-inflammatory (NSAIDs) are some of the most commonly prescribed medicines used worldwide. Although of undoubted efficacy for the treatment of musculoskeletal injury, it is well established that chronic administration of NSAIDs causes both gastric and intestinal damage. This
Abbreviations used in this paper: BSA, bovine serum albumin; EGF, epidermal growth factor; IGF, insulin like growth factor; NSAID, non-steroidal anti-inflammatory drug; TGF, transforming growth factor. 
(IGF-I) and IGF-II, each at about $2 \mathrm{mg} / \mathrm{l}$, transforming growth factor $\beta$ (TGF $\beta$ ) at 25 $\mu \mathrm{g} / \mathrm{l}$, and epidermal growth factor (EGF) at 6 $\mu \mathrm{g} / \mathrm{l}$ (data supplied by Scientific Hospital Supplies, personal communication).

\section{IN VIVO STUDIES}

Rat gastric damaging model

The ability of colostrum and milk to prevent gastric damage in rats stressed by indomethacin and restraint was assessed using our previously validated model. ${ }^{78}$ Rats were randomised to receive $2 \mathrm{ml}$ saline (containing bovine serum albumin (BSA) $0.2 \mathrm{mg} / \mathrm{ml}$ ), colostrum, or milk at $25 \%$ and $50 \% \mathrm{vol} / \mathrm{vol}$ or $12.5 \mathrm{ng}$ of recombinant $\mathrm{TGF} \beta_{1}$ made up in saline and BSA. This dose of TGF $\beta_{1}$ was chosen as it is similar to the total amount of TGF $\beta$ administered to animals who received the colostrum at $25 \% \mathrm{vol} / \mathrm{vol}$. All solutions also contained $2 \%$ hydroxymethylpropylcellulose to reduce the rate of gastric emptying. Thirty minutes after gavage, all rats received indomethacin (20 $\mathrm{mg} / \mathrm{kg}$ subcutaneously) and were placed in Bollman type restraint cages. Animals were killed three hours later and their stomachs removed and inflated with $4 \mathrm{ml}$ of $10 \%$ formalin. The stomachs were randomly coded and macroscopic and microscopic assessment of injury were assessed in a blinded fashion. Macroscopic injury was assessed using a dissecting microscope $(\times 10)$ with the aid of a reference square grid and reported as the total area of ulceration per stomach $\left(\mathrm{mm}^{2} /\right.$ stomach $)$. The stomachs were then embedded in wax and the depth of damage assessed microscopically as previously described. ${ }^{7}$ Briefly, microscopic injury was graded with a score from 0 to 4 where: $0=$ no damage, $1=$ one small erosion (less than $0.5 \mathrm{~mm}), 2=$ two small or one large erosion (greater than $0.5 \mathrm{~mm}$ ), $3=$ two or more large erosions, and $4=$ any area of ulceration extending to the muscularis mucosa.

\section{Mouse small intestinal injury model}

Protocol-Mice were randomised into groups of 20 and fed on a standard chow diet ad libitum. The drinking water was supplemented with $5 \%$ and $10 \%$ solution of colostrum or milk preparations for six days. Pilot studies showed that the addition of these preparations did not affect the total volume drunk (mean $5 \mathrm{ml} /$ mouse/ day). Small intestinal injury was induced in half of the animals of each group by administering a single dose of indomethacin $(85 \mathrm{mg} / \mathrm{kg}$ subcutaneously) 24 hours before the end of the study. Animals were killed 24 hours after indomethacin as we have previously shown that this is the time of maximal damage. ${ }^{9}$ In order to assess changes in proliferation, each animal also received vincristine $(1 \mathrm{mg} / \mathrm{kg}$ intraperitoneally), to induce metaphase arrest, two hours prior to killing.

Assessment of damage and proliferation-The wet weights of the various sections of the gastrointestinal tract were recorded. Samples of the small intestine and colon (defined by their percentage length) were fixed in Carnoy's fluid and stored in 70\% (vol/vol) ethanol. Quantitation of intestinal proliferation and vil- lus morphology (using microdissected villi) were determined using previously published methods. $^{9}$

\section{IN VITRO STUDIES} Cell proliferation

Rat intestinal epithelial (RIE-1) cells were a gift from $\mathrm{K}$ Brown, Babraham Institute, Cambridge, UK, and the human colonic carcinoma cells (HT-29) were obtained from ECACC, Porton Down, UK. RIE-1 and HT-29 cells were grown in Dulbecco modified Eagle medium (DMEM) containing glutamine and $10 \%$ foetal calf serum. Effects of colostrum, milk, and EGF (positive control) were subsequently tested under serum starved conditions. In addition, some wells also received TGF $\beta_{1}$ or a panspecific anti-TGF $\beta$ polyclonal antibody (R\&D Systems, Minneapolis, USA). To assess the rate of DNA synthesis, $\left[{ }^{3} \mathrm{H}\right]$ thymidine $(2$ $\mu \mathrm{Ci} /$ well) was included 12 hours after the addition of the test factors and cells were left for a further 12 hours. For each condition, the stimulatory or inhibitory effect of the solutions was measured in quadruplicate in four separate wells. Cell viability, determined by the ability to exclude $0.2 \%$ trypan blue, was greater than $90 \%$ in both RIE-1 and HT-29 cell lines.

\section{Cell migration as a model of wound repair}

RIE and HT-29 cells were grown to confluence in six well plates under the same conditions as for the proliferation studies. The monolayers were then wounded by scraping a disposable pipette tip across the dishes, washed with fresh serum free medium, and cultured in serum free medium in the presence of various doses of colostrum. The rate of movement of the anterior edges of the wounded monolayers was then determined by taking serial photomicrographs at various times after wounding. ${ }^{8}$ An inverted microscope (Nikon NK2) and a NIKON H3 camera with 100-fold magnification were used to obtain photomicrographs. Identical regions were examined at each time point by premarking the base of the plates to facilitate alignment. Twenty measurements per field were performed by placing a transparent grid over the photograph and measuring the distance moved from the original wound line. All results are expressed as mean (SEM) of four separate experiments.

\section{STATISTICS}

Studies were assessed using one way analysis of variance (ANOVA) for rat gastric damaging studies. Two way ANOVA was used for mouse small intestinal studies (factors: diet and presence of indomethacin). One or two way ANOVA were also used for the cell culture studies as appropriate. Where a significant effect was seen $(p<0.05)$, individual comparisons between groups were performed based on the group means, residual, and degrees of freedom obtained from the ANOVA, a method equivalent to repeated measures analysis. 

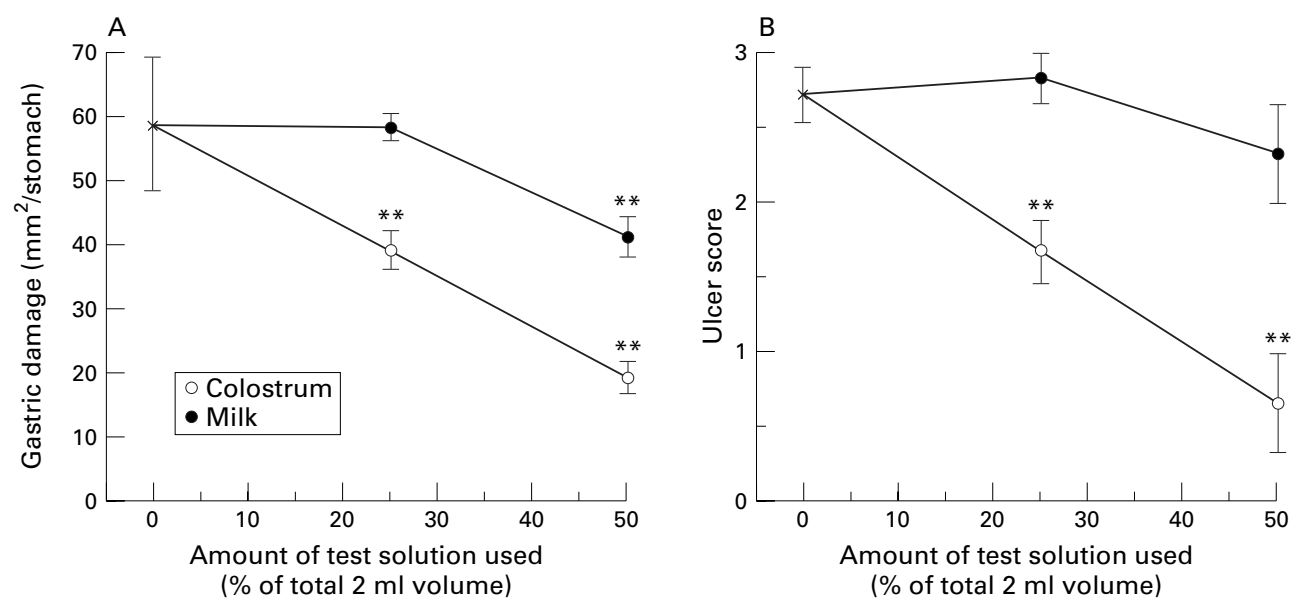

Figure 1 Effect of gavage with defatted colostrum and milk on the degree of gastric injury caused by indomethacin. The degree of injury was assessed macroscopically $(A)$, expressed as $\mathrm{mm}^{2} /$ stomach and microscopically (B), where microscopic injury was graded with a score from 0 to 4 . Results expressed as mean (SEM) of six rats per group. ${ }^{\star *} p<0.01$ versus amount of injury seen in animals receiving control solution.

\section{Results}

IN VIVO STUDIES

Gastric injury model

Colostrum caused a dose dependent reduction in the amount of gastric injury resulting from indomethacin and restraint (fig 1A). Milk solution was much less efficacious. Animals that received $12.5 \mathrm{ng}$ of recombinant $\mathrm{TGF} \beta_{1}$ instead of the colostrum also had a reduction in gastric injury of about $60 \%$ (macroscopic injury score 17 (4) $\mathrm{mm}^{2} /$ stomach versus 59 (10) $\mathrm{mm}^{2} /$ stomach in control animals, $\mathrm{p}<0.01)$. This dose of TGF $\beta_{1}$ was chosen as it is similar to the total TGF $\beta$ content in $25 \%$ $\mathrm{vol} / \mathrm{vol}$ colostrum. Assessment using the microscopic scoring system gave similar results (fig 1B).

\section{Small intestinal injury model}

Morphometry - Compared with controls, supplementation with colostrum or milk had no effect on villus height in animals who did not receive indomethacin. At both the jejunal and ileal sites, indomethacin caused a $25 \%$ reduction in the villus heights of control animals $(p<0.01$; fig 2). Similar changes were seen in animals which had received the $5 \%$ or $10 \%$ milk or the $5 \%$ colostrum solution. Animals

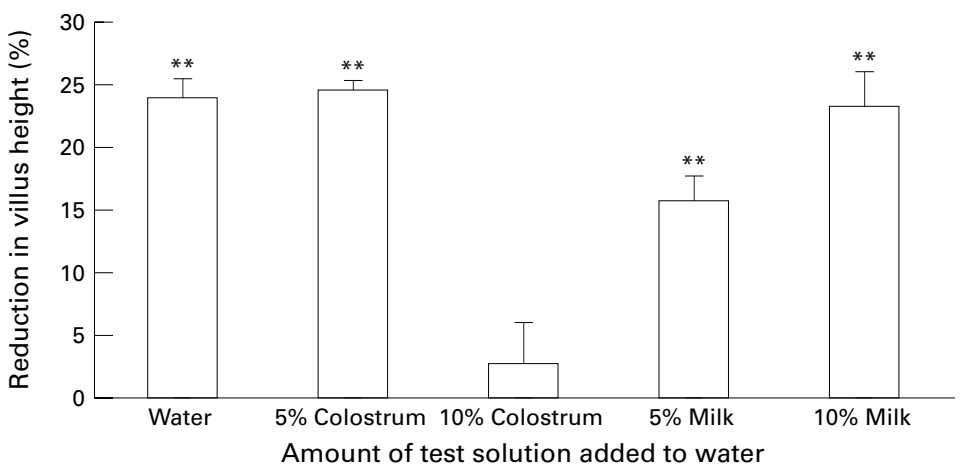

Figure 2 Effect of defatted colostrum and milk on the degree of small intestinal injury caused by indomethacin. Data are expressed as the percentage reduction in villus height of indomethacin treated animals compared with animals given the equivalent supplementation but no indomethacin. Results shown are those obtained from the jejunum and are expressed as mean (SD). ${ }^{\star *} p<0.01$ versus villus height in animals given the equivalent supplementation but no indomethacin. receiving $10 \%$ colostrum, however, had only about a $5 \%$ reduction in their villus height (NS; fig 2).

Metaphases-The feeding of colostrum or milk solution caused a small (10\%), but significant, reduction in the number of metaphases seen in the small intestine with a further reduction $(10 \%)$ seen when indomethacin was coadministered (at $30 \%$ small intestinal site, significance of the effect of diet was $\mathrm{p}=0.001$; effect of indomethacin, $p=0.002$; with no interaction, $p=0.72$ ). This means that the presence of colostrum or milk did not influence the amount of metaphase reduction induced by the indomethacin in any of the groups.

In the colon, the addition of indomethacin caused a $40 \%$ increase in the accumulation of metaphases per crypt which was unaffected by the presence of colostrum or milk supplementation (at the $25 \%$ colon site, significance of the effect of colostrum was $p=0.611$; effect of indomethacin, $\mathrm{p}<0.001$; interaction, $\mathrm{p}=0.25$ ).

\section{IN VITRO STUDIES}

Cell proliferation

HT-29 and RIE-1 cells showed a dose dependent bell shaped increase in thymidine uptake in response to colostrum, with the maximal effect seen when added at $30 \% \mathrm{vol} / \mathrm{vol}$, resulting in a threefold increase in thymidine uptake. For the dose response study utilising RIE-1 cells, uptake increased from 19900 (300) cpm under basal circumstances to 59200 (900) $\mathrm{cpm}$ when incubated in the presence of colostrum at $30 \% \mathrm{vol} / \mathrm{vol}(\mathrm{p}<0.01)$. In the subsequent series of experiments utilising size exclusion separation, this effect was found to be mainly due to factor(s) with molecular weights greater than $30 \mathrm{kDa}$ with virtually all the prostimulatory activity being found in this fraction (fig 3). Addition of pure TGF $\beta_{1}$ to these cell lines resulted in either no effect on proliferation or a small reduction in proliferation (data not shown), showing that the proproliferative effect of colostrum on these cell lines was not due to its TGF $\beta$ content. 


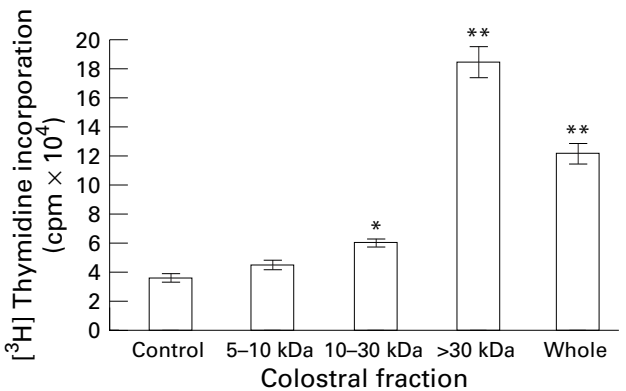

Figure 3 Effect of defatted colostrum on cell proliferation in RIE-1 cells. Results expressed as mean (SEM).

${ }^{\star} p<0.05,{ }^{*} p<0.01$ versus cells grown without colostrum.

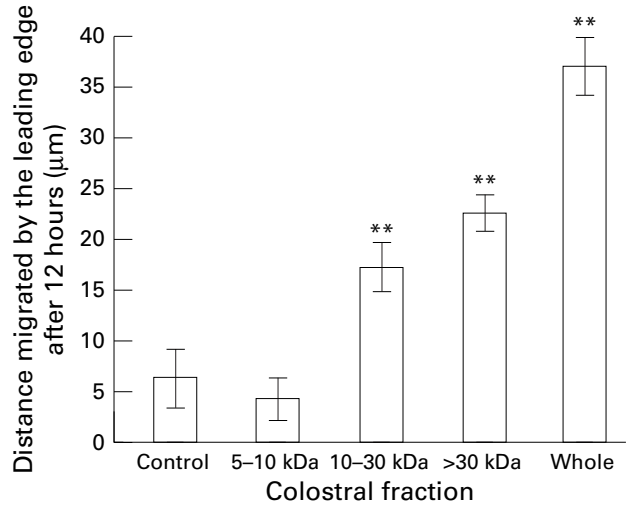

Figure 4 Effect of defatted colostrum on HT-29 cell migration, used as an in vitro model of wound repair. $\star_{*}^{*} p<0.01$ versus distance travelled by cells grown in control solution. Values expressed as mean (SEM).

Cell migration as a model of wound repair Addition of colostrum to HT-29 cells or RIE-1 cells resulted in a dose dependent increase in cell migration. For the HT-29 cells, maximal stimulation was seen at $30 \% \mathrm{vol} / \mathrm{vol}$, resulting in a fourfold increase in migration. Similar results were seen with the RIE-1 cell line. Size exclusion studies showed that predominant promigratory activity was present in both the $10-30 \mathrm{kDa}$ (accounting for about $40 \%$ of the effect of adding whole colostrum) and the $>30$ $\mathrm{kDa}$ fractions (accounting for about $60 \%$ of the effect of adding whole colostrum; fig 4). These percentage activities were similar in both cell lines.

\section{Discussion}

We have used several well validated in vivo and in vitro models to investigate the potential value of defatted milk and colostrum preparations in reducing NSAID induced gastrointestinal damage. Animal models showed that gastric and small intestinal injury caused by indomethacin could be reduced by colostrum and that a similarly prepared milk solution was far less efficacious.

For the in vitro studies, rat small intestinal (RIE-1) and human colonic (HT-29) cells were used to show that these effects were not species specific and because administration of NSAIDs causes damage throughout the entire gastrointestinal tract. Studies examining the potential beneficial effect of colostrum on gastric injury were performed using rats as we have previously validated this model for other growth factor studies (for example, Playford et $\left.a l^{8}\right)$. Similarly, mice were used for studying the effect of colostrum on indomethacin induced small intestinal injury as we have previous experience of using this model to assess effects of other regulatory peptides. ${ }^{9}$ In addition, we have found that mouse tissue is much easier to process and microdissect than the equivalent rat tissue (unpublished observation).

Colostrum is the milk produced by the mother for the first few days after birth and is much richer in growth factors and antibodies than ordinary milk..$^{5610}$ Previous studies have mainly examined the effect of colostrum on neonatal gut development; for example, porcine colostrum increases gut growth in suckling pigs. ${ }^{11}$ There has been little research, however, into the potential value of using milk or colostral fractions for adult gastrointestinal conditions. Bovine colostrum preparations are currently available in the USA, UK, and the rest of Europe as "over the counter" health food supplements. They do, however, contain large amounts of potent growth factors which we have shown are capable of influencing cell growth and migration in vitro and reducing indomethacin induced gut injury in vivo.

When an acute mucosal injury occurs, the initial phase of the repair process is the rapid migration of surviving cells over the denuded area, to reestablish a continuous epithelial layer. This begins within the first hour following injury and is termed "restitution". It is followed by a much slower increase in cell proliferation and remodelling. ${ }^{12}$ Our in vitro studies showed that the colostrum preparation studied was able to stimulate both migration and proliferation. Size exclusion studies showed that the majority of the biological activity involved in stimulating cell migration and proliferation in vitro could be found in the $>30 \mathrm{kDa}$ fraction. Epidermal growth factor and transforming growth factor $\alpha$ can both mediate increases in proliferation and migration; however, both have a molecular weight of about $6 \mathrm{kDa}$ and are therefore too small to account for our results. TGF $\beta$ is present in colostrum in a high molecular weight form (about $80 \mathrm{kDa}$ ) which is converted to a lower molecular weight form (about $25 \mathrm{kDa}$ ) when it comes into contact with an acidic environment $(\mathrm{pH}<2)$, such as gastric juice. ${ }^{13}$ Although TGF $\beta$ is known to stimulate restitution of intestinal epithelial cells, ${ }^{14}$ it is unlikely to be relevant to our finding of increased proliferation as the present studies have shown that addition of recombinant TGF $\beta_{1}$ had either no effect or a slight inhibitory effect on growth. The identities of the large molecule(s) responsible for the proproliferative effects of bovine colostrum are unclear but may include bovinecolostrum derived growth factor. The structure of this molecule is incompletely determined but it is known to have a molecular weight of about $30-35 \mathrm{kDa}$, and be structurally related to platelet derived growth factor; it has been shown to stimulate proliferation of mouse fibroblast $3 \mathrm{~T} 3$ cells. ${ }^{15} 16$ In contrast to the results from the in vitro work, our in vivo studies suggested that members of the TGF $\beta$ 
family were key components of the colostrum in its ability to prevent gastric injury in the rat model. Administration of recombinant TGF $\beta_{1}$, when given alone, reduced injury to a similar extent to that which occurred after administering colostrum. Studies employing neutralising antibodies could potentially shed light on which are the major peptides involved in these responses. There are, however, very limited data about the cross reactivity of commercially available antibodies (usually raised against human or murine forms) against the bovine homologues, seriously limiting the interpretation of such experiments. In addition, it is likely that more than one growth factor is important in these responses.

Non-steroidal anti-inflammatory drugs are of undoubted benefit for the treatment of musculoskeletal injury and are widely used; approximately 13 million patients in the USA regularly take NSAIDs for arthritic conditions ${ }^{17}$ and a prevalence study from Sweden found $8 \%$ of the total population were taking NSAIDs. ${ }^{18}$ However, $2-4 \%$ of patients who take an NSAID for a year develop serious gastrointestinal complications including peptic ulceration and bleeding from the stomach and small intestine. ${ }^{19-21}$ Many of these complications develop in patients who have no warning symptoms and elderly patients taking NSAIDs are at particular risk. Current therapeutic options include coadministration of damage limiting drugs, particularly acid suppressants and prostaglandin analogues or using the relatively selective (and expensive) COX-II inhibitors. However, none of these options are optimal; acid suppressants and prostaglandin analogues are able to reduce gastric injury but are relatively inefficient in preventing small intestinal damage, as shown by studies which found that the major increase in small intestinal permeability caused by indomethacin could only be partially reduced by coadministration of synthetic prostaglandins. ${ }^{3}$ In addition, prostaglandin analogues induce diarrhoea in a proportion of patients and are relatively contraindicated in women of child bearing potential because of their proabortive and teratogenic properties. ${ }^{4}$ The COX-II inhibitors currently available are not completely selective and are associated with gut injury, ${ }^{22}$ particularly in patients requiring higher doses. ${ }^{23}$ All of the present therapeutic options are therefore suboptimal and new approaches are required.

Indomethacin causes damage to the gastrointestinal tract by several mechanisms including reduction of mucosal prostaglandin levels, reduction of mucosal blood flow, stimulating neutrophil activation, and possibly also stimulating apoptosis. ${ }^{24}$ It is likely that many of these mechanisms will be influenced by the numerous growth factors present in the colostrum preparation. We have previously shown that several of these peptides, for example, EGF and TGF $\alpha$, are susceptible to digestion from luminal proteases when administered alone $e^{25}$ and that peptides involved in mucosal repair can act in a synergistic fashion if coadministered. ${ }^{26}$ There are therefore several reasons why the use of this preparation, as opposed to giving a single recombinant peptide, might be particularly beneficial. It is also important to note that our studies show that the division between "food products" and "drugs", when considered in terms of biological activity, is far from clear. The colostrum preparation used for these studies is currently considered to be a food product for licensing purposes. Based on our results, we consider that products such as this should be considered as "nutriceuticals" as they contain potent biologically active molecules.

In summary, we have shown that this colostrum preparation has major beneficial effects in preventing NSAID induced gut injury in a variety of in vivo and in vitro models. Further studies are underway to determine its value in patients taking long term NSAIDs and in the treatment of other ulcerative conditions of the bowel, such as necrotising enterocolitis and inflammatory bowel disease, where therapy is suboptimal and novel approaches are required.

The authors acknowledge the Wellcome Trust, Medical Research Council, and Scientific Hospital Supplies for funding.

1 Allison MC, Allan G, Howatson AG, et al. Gastrointestinal damage associated with the use of non-steroidal antiinflammatory drugs. $\mathrm{N} \mathrm{Engl} \mathrm{F} \mathrm{Med} \mathrm{1992;327:749-54.}$

2 Bjarnason I, Zanelli G, Smith T, et al. Non-steroidal anti-inflammatory drug induced intestinal inflammation in humans. Gastroenterology 1987;93:480-9.

3 Bjarnason I. Non-steroidal anti-inflammatory drug induced small intestinal inflammation in man. In: Pounder R, ed. Recent advances in gastroenterology. Vol 7 . London: ChurchRecent advances in gastroenterology.

4 Pastuszak AL, Schuler L, Speck-Martin CE, et al. Use of misoprostol during pregnancy and Mobius' syndrome in misoprostol during pregnancy and Mob
infants. N Engl $\mathcal{F}$ Med 1998;338:1881-5.

$5 \mathrm{Xu}$ RJ. Development of the newborn GI tract and its relation to colostrum/milk intake: a review. Reprod Fertil Dev 1996;8:35-48.

6 Xanthou M, Bines J, Walker WA. Human milk and intestinal host defence in newborns: an update. In: Advances in paediatrics. Vol 42. Moseby Year Book Inc., 1995:171-200.

7 Playford RJ, Vesey DA, Haldane S, et al. Dose dependant effects of fentanyl on indomethacin induced gastric damage. Digestion 1991;49:198-203.

8 Playford RJ, Marchbank T, Chinnery R, et al. Human spasmolytic polypeptide is a cytoprotective agent which stimu-
lates cell migration. Gastroenterology 1995;108:108-16.

9 Playford RJ, Marchbank T, Goodlad RA, et al. Transgenic mice which overexpress the human trefoil peptide pS2, mice which overexpress the human trefoil peptide $\mathrm{pS} 2$,
have an increased resistance to intestinal damage. Proc Natl Acad Sci 1996;93:2137-42.

10 Steimer KS, Packard R, Holden D, et al. The serum free growth of cultured cells in bovine colostrum and in milk obtained later in the lactation period. If Cell Physiol 1981;109:223-34.

11 Simmen FA, Cera KR, Mahan DC. Stimulation by colostrum or mature milk of gastrointestinal tissue development in newborn pigs. F Anim Sci 1990;68:3596603.

12 Svanes K, Itoh S, Takeuchi K, et al. Restitution of the surface epithelium of the in vitro frog gastric mucosa after damage with hypermolar sodium chloride. Gastroenterology 1982;82:1409-26.

13 Rogers M-L, Goddard C, Regester GO, et al. Transforming growth factor $\beta$ in bovine milk: concentration, stability and growth factor $\beta$ in bovine milk: concentration, stability
molecular mass forms. F Endocrinol 1996;151:77-86.

14 Dignass AU, Podolsky DK. Cytokine modulation of intestinal epithelial cell restitution: central role of transforming nal epithelial cell restitution: central role of transformi

15 Shing YW, Klagsbrun M. Purification and characterisation of a bovine colostrum-derived growth factor. Mol Endocrinol 1987;1:335-8

16 Shing YW, Klagsbrun M. Human and bovine milk contain different sets of growth factors. Endocrinology 1984;115: 273-82.

17 Fries JF. NSAID gastropathy: the second most deadly rheumatic disease? Epidemiology and risk appraisal. f Rheumatol 1991;28(suppl):6-10.

18 Wilholm BE, Myrhed M, Ekman E. Trends and patterns in adverse drug reactions to non-steroidal anti-inflammatory drugs reported in Sweden. In: Rainsfoird KD, Velo GP, eds. Side effects of anti-inflammatory drugs. Lancaster: MTP Side effects of ant
press, 1987:55-70.

19 MacDonald TM, Morant SV, Robinson GC, et al. Association of upper gastrointestinal toxicity of nonsteroidal anti-inflammatory drugs with continued exposure: cohort study. BMf 1997;315:1333-7. 
20 Paulus HE. FDA Arthritis Advisory Committee Meeting: Serious gastrointestinal toxicity of non-steroidal anti-
inflammatory drugs; drug containing renal and biliary inflammatory drugs; drug containing renal and biliary
stones; diclofenac and carpofen approved. Arthritis Rheum stones; diclofenac

21 Langman MJS, Morgan L, Worrall A. Use of antiinflammator large bowel perforations and haemorrhage. BM7 1985;290 $347-9$.

22 Wojtulewski JA, Schattenkirchner M, Barcelo P, et al. A six month double blinded trial to compare the efficacy and safety of meloxicam $7.5 \mathrm{mg}$ daily and naproxen $750 \mathrm{mg}$ daily in patients with rheumatoid arthritis. Br $\mathcal{F}$ Rheumatol 1996;35(suppl 1):22-8.
23 Distel M, Mueller C, Bluhmki E, et al. Safety of meloxicam-a global analysis of clinical trials. $B r f$ Rheumatol 1996;35:68-77.

24 Levi S, Shaw-Smith C. Non-steroidal anti-inflammatory drugs: how do they damage the gut? Br f Rheumatol 1994; 33:605-12.

25 Playford RJ, Watanaba P, Woodman AC, et al. Effect of luminal growth factor preservation on intestinal growth. luminal growth factor

26 Chinery R, Playford RJ. Rapid communication: combined intestinal trefoil factor and epidermal growth factor is prophylatic against indomethacin-induced gastric damage in the rat. Clin Sci 1995;88:401-3. 